\title{
Narrar-nos es formar-nos: las historias de vida en la formación de maestros
}

TO NARRATE OURSELVES IS TO TRAIN OURSELVES: LIFE STORIES IN TEACHER TRAINING

n NARRAR É FORMAR-NOS: AS HISTÓRIAS DE VIDA NA FORMAÇÃO DOS PROFESSORES

Mireya González Lara* / mireyagonzalezlara@fundacionconvivencia.org

\section{Resumen}

El presente artículo explora la relación entre las historias de vida y la formación docente a través de tres experiencias formativas realizadas entre el año 2010 y el 2014; además de mostrar la manera cómo se configura esta relación, se avanza en las posibilidades formativas de las historias de vida y en los efectos de la historia de vida en la formación.

\section{Abstract}

This article explores the relationship between life stories and teacher training through three training experiences carried out between 2010 and 2014. In addition to showing how this relationship is formed, it examines the educational possibilities of life stories and the effects of life stories in teacher training.

\section{Resumo}

O presente artigo explora a relação entre as histórias de vida e a formação docente através de três experiências formativas realizadas entre os anos 2010 e 2014; além de mostrar a maneira como essa relação é configurada, avança nas possibilidades formativas das histórias de vida e nos efeitos da história de vida na formação.
Palabras clave

Historia de vida, formación docente, sujeto, maestro, narración

\section{Keywords}

Life story, teacher training, subject, teacher, narrative.

\section{Palavras chave}

Histórias de vida, formação docente, sujeito, professor, narração

Asesora Fundación Convivencia, Magíster en Historia, Pontificia Universidad Javeriana

Fecha de recepción: 20 de abril de 2016 / Fecha de aprobación: 26 de mayo de 2016 


\section{Introducción}

La fuerza de la formación docente en las urgencias de la política educativa actual no deja de causar sospechas. Este retorno no necesariamente llega cargado de nuevas concepciones o perspectivas de trabajo; la formación de docentes, desde los años sesenta y setenta del siglo xx ha estado inscrita en una amplia y profunda tradición de producción de conocimiento que ha configurado el escenario escolar, el saber pedagógico y al sujeto maestro, en convenciones asociadas a la reproducción de conocimiento, a la subordinación a otros especialistas y a la instrumentación de una práctica. El presente artículo recoge tres experiencias de formación que introdujeron y exploraron alternativas formativas a docentes, tomando las historias de vida como uno de sus ejes y haciendo de la historia su "contenido", su metodología y una finalidad de esta 1 .

\section{Trayectos de una relación: historias de vida y formación de docentes}

Las historias de vida, las autobiografías o los ejercicios narrativos en los procesos de formación a docentes no tienen una larga trayectoria en nuestro contexto. En el balance realizado en torno a la formación docente y las historias de vida durante el periodo comprendido entre 1990 y 2010 (González, Jiménez, Neira y Cortes), se subraya la emergencia de esta relación asociada con el retorno al sujeto en los paradigmas científicos e investigativos del Ilamado giro epistemológico de las ciencias sociales sucedido en los años sesenta y setenta del siglo xx. La introducción de las historias de vida en la investigación del campo de la educación es su antecedente más inmediato, e interpela los supuestos del carácter "científico"; especialmente, en términos de la relación entre el sujeto investigador y el objeto investigado.

A diferencia de otros campos, este giro solo comenzó a impactar los ámbitos de la formación de maestros dos o tres décadas luego de haberse instalado en los

1 Las tres experiencias son las siguientes: 1) Diplomado en Enseñanza de la Historia en el marco del Bicentenario, Bogotá D.C., 2010; 2) ¿El puente está quebrado? Acompañamiento a docentes noveles en Bogotá. Bogotá D. C., 2013-2014, y 3) Movimiento Pedagógico, memoria y maestros, Bogotá, 2013. Los tres procesos fueron diseñados y desarrollados en alianza con la Organización de Estados Iberoamericanos (OEI), la Secretaría de Educación del Distrito Capital (SED) y el Instituto para la Investigación Educativa y el Desarrollo Pedagógico (IDEP). paradigmas de la investigación. Una posible explicación de este tardío impacto podría relacionarse con la tradición en la que se funda la formación de maestros, instalada en el cruce entre las perspectivas positivistas de la producción del conocimiento y el sólido consenso social y político en torno a la función reproductivista de la escuela. Esta última, como institución de saber, no escapa de las formas de organización social y política de la producción del conocimiento, y produce efectos que alcanzan a impactar la naturaleza del saber de la enseñanza y al sujeto portador de dicho saber: la pedagogía y el maestro.

Es interesante constatar el impacto del giro epistemológico en los procesos investigativos de las instituciones de educación superior (incluso, en las formadoras de maestros), al generar otros paradigmas $\mathrm{o}$, por lo menos, interpelar críticamente los existentes; no sucedió lo mismo en las concepciones ni las prácticas de formación de maestros de estas instituciones: por el contrario, se profundizó la brecha entre investigación y formación. La formación siguió siendo un proceso "técnico" en el que el sujeto recibía "transformado", o, en el mejor de los casos, para transformar aquello que debía enseñar.

Los trabajos en el campo de la enseñanza o sobre la identidad docente rastreados en el mundo anglosajón desde la década de 19802 fueron los primeros ejercicios en convocar perspectivas investigativas centradas en los sujetos, en la enseñanza o en el aprendizaje. Inicialmente con propósitos asociados a la mayor efectividad del aprendizaje de los estudiantes, estas investigaciones se constituyeron en una importante veta de indagación que fue $y$ ha sido explorada largamente y con diferentes perspectivas e intencionalidades. Cabe subrayar que para los años ochenta del siglo xx, un grupo amplio de investigadores en educación, procedentes en especial de la sociología y de la antropología en Estados Unidos e Inglaterra, ya afirmaban que "Para entender algo que es tan intensamente personal como lo es la enseñanza, es crucial conocer qué clase de persona es el maestro". (Goodson, 1981, p. 69).

\footnotetext{
2 Ver: Goodson, I. (1981). Life history and study of schooling. Interchange. Institute for Studies in Education. Ontario, Canada; Goodson, I. (1994). Studying the teachers' lives. Professional Life and Work. Routledge. Gran Bretaña; Goodson, I. (2004). Historias de vida del profesorado. Barcelona, España: Octaedro.
} 
A la vez que las investigaciones producían cambios en las formas de entender la enseñanza y en los saberes asociados a ella y al sujeto responsable de ella, también se dejaba constancia sobre la incidencia del sujetomaestro en la construcción de un saber que se hacía en la práctica. El cambio en "(...) la percepción del maestro como un simple instrumento en la producción de logros académicos a verlo como un agente inteligente en la educación de los niños" (Goodson, 1994, citado por Clavijo, 2000, p. 19) fue gracias, entre otras razones, a la introducción del saber biográfico en la investigación, y, posteriormente, a los procesos de investigación en los que fueron implicados los maestros para su formación, mientras que para el contexto anglosajón esta perspectiva ya era documentada desde el decenio de 1980 en la formación de los maestros bilingües, y luego, en la formación con perspectiva de género y en el acompañamiento a los docentes noveles; para el mundo iberoamericano (especialmente, en Argentina y España), la emergencia de las historias de vida en procesos formativos se sucede durante las postrimerías del milenio, asociada, principalmente, a los procesos formativos de docentes noveles ${ }^{3}$.

El retorno al sujeto, como eje de la investigación cualitativa, y posteriormente, de la formación, puede asociarse al amplio consenso que ha ganado en la educación la idea de la construcción del conocimiento, que reconoce el plano intersubjetivo de la realidad social. Esta perspectiva cobró importancia en el mundo académico durante las últimas décadas del siglo xx, y

3 Ver, entre otros: Alliaud, A. y Suárez, D. (2011). El saber de la experiencia: narrativa, investigación y formación docente. Clacso. Facultad de Filosofía y Letras de la Universidad de Buenos Aires. Alliaud, A. y Vezub, L. (2012). El acompañamiento pedagógico como estrategia de apoyo y desarrollo profesional de los docentes noveles. Aportes conceptuales y operativos para un programa de apoyo a los docentes principiantes en Uruguay. Montevideo, Uruguay: OEI-Ministerio de Educación y CulturaConsejo de Formación en Educación-Aecid. Allen, B., Alliaud, A. et al. (2013). Desarrollo profesional de formadores para el acompañamiento pedagógico de docentes noveles. Aportes, dispositivos y estrategias presentadas en el programa de Formación de Formadores de Uruguay. Montevideo, Uruguay: OEI-Ministerio de Educación y Cultura-Consejo de Formación en Educación-Aecid. Knowles, J. (2005). Modelos para la comprensión de las biografías del profesorado en formación y en sus primeros años de docencia". Historias de vida del profesorado. Barcelona, España: Octaedro. Lucas, M. (1986). El proceso de socialización: Un enfoque sociológico. Revista Española de Pedagogía 173, 357-370. Marcelo, C. (1991). Aprender a enseñar. Un estudio sobre el proceso de socialización de profesores principiantes. Madrid: CIDE. Marcelo, C. (2009). Los comienzos en la docencia: un profesorado con buenos principios. Revista del Curriculum y Formación del Profesorado 13 (1). produjo cambios tanto en los objetos de la investigación como en sus formas de proceder.

Las trayectorias investigativas en la educación para 1980, tanto en el mundo anglosajón como en el iberoamericano, ya mostraban una ruptura en cuanto a las concepciones sobre el sujeto-maestro. Durante los años sesenta del siglo xx,

(...) las figuras de los maestros eran figuras insubstanciales en el panorama educativo que sobresalían, o se conocían, por medio de encuestas hechas a gran escala, o por análisis históricos de su posición en la sociedad; el concepto clave que se manejaba para entender la práctica docente era el de rol. (Ball y Goodson, 1985, p. 6, citados por Clavijo, 2000, p. 20).

A finales de los años sesenta y principios de los setenta, los investigadores (especialmente, los de estudios de caso) empezaron a ver las complejidades que atañen a la enseñanza como un proceso social y cultural; sobre todo, en consideración a las diferencias entre los alumnos. Finalizando el decenio de 1970, ante la hegemonía de los currículos y de la tecnología educativa, se observó otro cambio:

la atención se empezó a dirigir hacia las limitaciones que tenían los docentes en su trabajo (...) los maestros se transformaron de 'villanos' en 'víctimas' y en algunos casos, en 'víctimas de engaños' del sistema en que debían operar. Pero esta última caracterización de los maestros finalmente desembocó en la pregunta, cómo ven los docentes su trabajo y sus vidas. (Goodson, 1994, pp. 30-31).

A pesar de estas rupturas, aún no se lograba superar el lugar del sujeto-maestro como objeto de estudio; entre otras razones, por el fuerte arraigo de la concepción instrumental de la enseñanza, cuya relación con la investigación se hallaba sujeta a finalidades prescriptivas asociadas a su posible incidencia en la eficiencia de los aprendizajes de los estudiantes; en menor medida, la investigación procuraba mejores comprensiones sobre el acto educativo y la enseñanza, y era más extraño aún encontrar investigaciones que tuvieran como principal objetivo la formación de los maestros. 
Será la investigación educativa, que comienza a vincular el análisis de la vida del maestro con su trabajo a través de la implementación de diferentes métodos biográficos, la que permitiría la ruptura; se comenzará a comprender la complejidad de la enseñanza, entre otros factores, por su naturaleza subjetiva. Ya no solo el maestro era un sujeto importante en la investigación, sino que su práctica era "(...) mucho más que las cosas técnicas que hacemos en el salón de clase -tiene que ver con quienes somos, con nuestra manera de vivir 'la vida'" (Goodson, 1994, p. 29).

La indagación por estos senderos permitió profundizar en perspectivas investigativas que inicialmente se especializaron en el uso de modalidades asociadas con el método biográfico, y posteriormente exploraron enfoques colaborativos donde se requería la interacción entre el maestro como investigador, y el investigador externo. El uso de métodos investigativos que comprometen las trayectorias vitales de los maestros para intentar comprender los contextos, los ámbitos, las prácticas o a los sujetos, así como la participación de los maestros en los procesos investigativos, se constituyeron en las rutas que permitieron, entre otras cosas, pensar la relación entre investigación y formación de maestros:

(...) se puede afirmar que las narraciones personales, las historias de vida y las autobiografías utilizadas en la formación de docentes según se presentan en la literatura son maneras muy importantes de recopilar el saber de los docentes y de entender sus prácticas y sus valores sociales, históricos y culturales. Las narraciones personales de los maestros también ofrecen información y herramientas para entender cómo su educación puede influir en la educación de futuras generaciones. (Clavijo, 2000, p. 22).

Desde estas posiciones empezaron a valorarse el escenario de la cotidianidad del sujeto y todo lo personal que la constituye: sus formas de deseo, su historicidad, sus relaciones, sus palabras y sus acciones. Poner en juego al sujeto en los procesos investigativos ha sido, necesariamente, una convocatoria a su experiencia, que se constituye en el ejercicio reflexivo de su práctica.

Cuando tratamos de pensar de manera reflexiva acerca de nuestras experiencias educativas con el propósito de esclarecer los asuntos pedagógicos centrales que están en juego, estamos tratando de poner en marcha un tipo de práctica reflexiva, o de reflexión autobiográfica. No en el sentido de desarrollar un proyecto de indagación que siguiera el método fenomenológico-hermenéutico; más bien, pretendiendo dar lugar a un tipo de experiencia de formación ("bildung"), en el sentido en que pensar de manera orientada hacia lo vivido, abre un camino de transformación personal que nos coloca otra vez en la acción de una manera más diligente. (Caparrós y Sierra, 2012, p. 63).

Podría afirmarse, entonces, que en tanto comienzan a transformarse las comprensiones sobre el tipo de saber del maestro, también empiezan a producirse cambios en sus procesos formativos; se avanza en entender que su saber es social y culturalmente situado, que no está dado previamente en su totalidad, sino que se construye de manera constante en la práctica, pues se produce mientras se enseña. Hoy se comprende mejor que en el saber de los maestros, seguramente, la teoría no siempre antecede a la práctica; que el conocimiento producido en la investigación es diferente del conocimiento que se produce para su enseñanza, y que el conocimiento práctico puede ser emocional, relacional y moral.

\section{Posibilidades formativas de la narración: las historias de vida}

Profundizar en las posibilidades reflexivas del sujeto y en su relación con los otros, como procesos fundamentales para la constitución del maestro, define el desplazamiento metodológico acontecido en las tres experiencias formativas a docentes que sirven de base para el presente documento (ver nota 1). Esta movilización fue evidente al transitar de una propuesta cimentada en un trabajo de orden biográfico a otra enmarcada dentro de la producción de historias de vida. Se transitó de un ejercicio enfocado, principalmente, en el rigor histórico que implicó la construcción de los acontecimientos —cronología y secuencialidad-y su contextualización con perspectivas sociales, políticas y económicas más amplias, hacia enfoques menos estructurados en las disciplinas, que priorizaban la conversación y el diálogo entre colegas, la creación esencialmente literaria y la democratización y la legitimación en la producción de narrativas. 


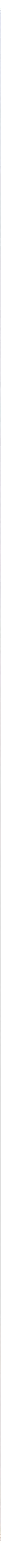


La historia de vida se constituyó en una metáfora para construir sentido sobre lo que le acontece al sujeto como maestro; fue una manera de abordarse en un momento y un lugar determinados hacia un horizonte de la experiencia, donde, justamente, se han jugado otro tipo de significados sobre lo vivido. Un presente, como un punto cero, que acoge un pasado cambiante y complejo, e incluyente de una visión sensible de todo lo que desde el hoy se considera que ha influido en la trayectoria de vida. La historia de vida cambia cuando se relata por otros, o bien, cuando se relata para otros; es inestable y compleja; a veces se silencia y a veces se oculta; se vuelve aprehensible cuando se escribe y se vuelve colectiva cuando se da lugar a otras voces, a través de polifonías que también van constituyendo al sujeto.

La historia de vida permite entenderse como finito, cambiante y eventual; también concede pensar la educación, nuevamente, como una transacción de lo humano, una posibilidad de transformarse a través de los otros. La enseñanza deja de ser un asunto exclusivamente técnico que atañe a la organización de un contenido para ser aprendido por otros, y se fue constituyendo en una práctica social y cultural en la que se afectan maestros y estudiantes, y, mutuamente, se producen como tales.

La historia de vida es un motivo de aprendizaje sobre lo que se es, lo que se ha dejado de ser y lo que se desea ser; sobre lo que se acoge y lo que se desecha; sobre las razones por las que mutan o permanecen los sueños y las pesadillas; sobre los límites y las posibilidades; sobre lo que se agota y lo que se renueva; sobre lo que moviliza y lo que paraliza; sobre los temores, los miedos y los intereses; sobre lo que los otros evocan o silencian en cada uno, $y$, seguramente, sobre lo que cada cual produce en los otros.

La construcción de las historias de vida comenzó mediante ejercicios centrados en una arquitectura de líneas temporales, cada vez más completas y complejas en cuanto a la cantidad de datos y acontecimientos y a sus posibles relaciones; y transitó a relatos, también rigurosos, donde un fragmento de estas trayectorias vitales se habría convertido en el foco de la mirada. Parecía como si se hubiera pasado de un relato de sí, a uno desde sí. Mientras que el primero tuvo una intencionalidad histórica y contextual, en el segundo, al desmarcarse de las temporalidades, el sujeto fue a la vez objeto y objetivo del proceso formativo; de manera pendular, osciló entre protagonista, testigo y biógrafo, como sucedió en el último de los procesos formativos.

El proceso de construcción de la historia de vida fue entendido como un ejercicio de segundo orden; o sea, como una lectura sobre el registro biográfico que permitía ubicar aquellos asuntos sobre los cuales se ocupaban los sujetos en ese momento. En torno a dicho asunto se volvía a organizar, a seleccionar y a estructurar una nueva narrativa que, por supuesto, en algunos casos abandonó, desbordó o superó el ejercicio biográfico inicial, sin el cual no habría sido posible esta nueva producción. Este segundo orden en la lectura organizó de otra manera los trayectos de vida, en tanto se privilegiaron los sentidos vitales que los sujetos dieron a las narrativas.

Cuando un sujeto se examina construye una narración que no procede únicamente de su capacidad de inventiva. "El sujeto no se inventa, si inventar es disponer de libertad absoluta para mirar hacia cualquier lado" ha planteado Gadamer (citado por Palou y Fons, 2012); los asuntos que lo ocupan en cada momento y cada lugar se constituyen en una especie de filtro por el cual pasa cada situación de su cotidianidad. Continuando con Gadamer, los sujetos no pueden dejar "pasar de largo", pues la mirada está en lo que le acontece, y abrirse, luego, eso sí, a las diversas interpretaciones. Entonces, "Comprender es dejarse Ilevar por aquello que queremos comprender, nunca dejarlo a un lado" (Palou y Fons, 2012, p. 181).

Esta exploración en torno a lo biográfico y a la historia de vida se constituyó en la reivindicación de la subjetividad, de las relaciones sujeto-sujeto y de lo narrativo: en unos casos, para dar cuenta de las relaciones de significado y de las construcciones simbólicas en la escuela; en otros, para hacer evidentes las formas de apropiación de los discursos; especialmente, los de la política educativa - los derechos, la infancia, la formación, la historia del país, etc.- - y también, para identificar las creencias y los valores de los maestros en la enseñanza, y en otros casos, como testimonio para suscitar el diálogo entre perspectivas particulares 
de los sujetos con procesos macrohistóricos de orden social y cultural. Su uso combinó las intencionalidades investigativas con los propósitos formativos, lo cual se constituyó en una de sus mayores riquezas.

Este giro hacia la narrativa, como elemento organizador de la experiencia y fundamental para dar cuenta de la manera como se abordan las relaciones de significado en el sujeto, se constituye en el eje que da cuenta de la manera como el sujeto se relaciona, interpreta y significa, y cuyo punto de llegada ha sido el redescubrimiento de su subjetividad, de la manera como se produce el sujeto. La experiencia solo puede existir en el acto de la narración cuando se transita del hablar sobre lo que pasa a relatar lo que nos pasa. Las experiencias no son previsibles; no permiten, a diferencia de la vivencia, la planificación, según Mélich (2012). Es la experiencia lo que genera la intriga, porque la respuesta nunca se puede prever con certeza. La experiencia, por lo tanto, nos interpela, y, como afirma Gadamer (citado por Palou y Fons, 2012) es en este momento, cuando algo nos interpela, que comienza la comprensión.

La experiencia del maestro, la experiencia de sí, le permite entenderse como un sujeto en proceso de ser sujeto, como un sujeto en el proceso de convertirse en maestro. La identidad no deja de ser sino la narración de cada sujeto, expresada en función del sistema cultural que se constituye constantemente a partir de su práctica. Lo que inicialmente se había propuesto como un proceso de construcción de la identidad, en términos biográficos y relacionales, como proyecto de sí y, a la vez, con y desde los otros, abrió paso a la identidad como construcción narrativa, en términos de Ricoeur (2006, p. 21).

La historia de vida expresa y valora la esencia subjetiva del sujeto, y en ella convergen diferentes dimensiones como la memoria, la cultura, la conciencia y la proyección de futuro, las cuales expresan la apropiación de la historicidad social, a la vez que le confieren sentido y animan su potencialidad (Zemelman, 1998). La subjetividad está presente en todas las dinámicas sociales y en todos los ámbitos de la vida cotidiana de la experiencia personal, y en este caso, de la formación de los maestros. La historia de vida como producción subjetiva es simultáneamente constitutiva del proceso social y se encuentra constituida por él, es producto y es productora de discursos y acciones. La historia de vida valora de manera positiva la experiencia del sujeto, las necesidades y las ataduras del presente, pero también se convierte en un elemento potencializador de futuro.

En el acto de narrar, de transitar del recuerdo a la palabra, de lo inestable y lo cambiante al registro y a la permanencia, de transformar el saber en decir (White, 1981), se expone al sujeto en su diálogo con el adentro y el afuera, con las voces del pasado, de la cultura y de la comunidad. Campos, citando a Bajtin, plantea:

El hombre no dispone de un territorio en el que es soberano. Siempre, mirándose a sí mismo descubre los ojos del otro o ve con los ojos de ese otro. El lenguaje es social en toda instancia expresiva, intersubjetivo, nunca neutro ni sin destinatario. Él es por naturaleza polifónico y se comunica en una amalgama de voces que tienen orígenes diversos. Somos 'nosotros', nunca el 'yo' individual y autónomo. (2012, p. 94).

En efecto, las experiencias formativas circularon entre los sujetos, los otros y lo colectivo; a la vez que se comprendían las diferentes narraciones en un sentido personal, social y cultural, también se subrayaba su carácter polifónico y público. Aunque las experiencias formativas transitaron metodológicamente por estos senderos, con diferentes estrategias y dinámicas, cada una de ellas hizo un énfasis particular: la primera, sobre los maestros, y la segunda, en la otredad.

Esta trayectoria en la formación no quiso ser lineal, acumulativa ni progresiva; es decir, no pretendió coherencia, o pasar de un relato a la concatenación de muchos relatos, o a producir mejores o más completos metarrelatos. El carácter polifónico y público de los ejercicios biográficos puso en evidencia las múltiples tensiones en las propuestas formativas, por ejemplo, la relación entre el sujeto y la historia, entre las subjetividades y las alteridades y entre lo colectivo y el sujeto.

Se comprendió mejor que la historia es un relato de naturaleza diferente de la naturaleza de la memoria, y que su relación se potencia en tanto se comprendan sus diferencias; que la memoria colectiva y la individual son afectadas, $y$, seguramente, transformadas, en tanto se individualizan y se colectivizan; que las biografías, como las obras de arte, no necesariamente dan cuenta 
de un momento histórico objetivo, por cuanto los mundos subjetivos de donde proceden tienen otros tiempos, espacios, secuencialidades y, con seguridad, otras formas de percibirlo. Se entendió, entonces, que cuando se relata, nada queda igual: ni los colectivos de donde proceden y adonde llegan los relatos, ni los sujetos que los producen y los asimilan; tampoco, las huellas que van siendo parte de las identidades. Los relatos mueven las maneras de representarse en el colectivo o en la individualidad.

\section{La formación tras las historias de vida}

La introducción de los enfoques biográficos y narrativos en la formación de maestros, sea como metodología, enfoque o intencionalidad, produjo la remoción de sus presupuestos básicos y sus prácticas convencionales. Particularmente, la intención de volver "sobre el sujeto" ( $y$, en especial, en torno a la idea de desmarcarse de su saber disciplinar) fracturó lo que, de entrada, ha justificado la formación continua de maestros. Los asuntos que circulan en torno a estas iniciativas interrogan, principalmente, la configuración de estos sujetos en términos de todo eso que ha "confabulado" a su favor o en su contra para ser maestro, y en su relación con el saber.

Un primer elemento fue identificar los saberes implicados en este oficio, la manera como se constituyen y sus formas de relación; en efecto, hay saberes acerca de la enseñanza, otros sobre lo que se enseña y otros más relacionados con el carácter ético, afectivo y estético de la enseñanza; es decir, con la "formación integral" de los sujetos. Los saberes que requiere el maestro para la enseñanza se dimensionan en la práctica, la cual acontece en los escenarios escolares situados, y caracterizados, generalmente, por ser muy demandantes de acciones comprensivas, creativas y, en lo posible, asertivas frente a cada una de las situaciones que a diario se presentan. La formación de maestros, entonces, es un ejercicio, acogiendo a Meirieu (1998), que se instala en la pedagogía; es decir, en ese espacio entre la ingeniería y la incertidumbre.

Un segundo elemento fue ubicar al sujeto-maestro como el eje de la formación, lo cual implicó un ejercicio de creciente complejidad, al irse haciendo evidente la fuerza que ejercen las múltiples y complejas tensiones que configuran el campo de las políticas educativas. Esta complejidad se acrecienta ante la permanente exigencia que le implica al sujeto ser maestro, en términos de su saber y de su conocimiento; también, en relación con su manera de ser, y, necesariamente, con la manera de entregarse a los demás; tal parece que una forma de constituirse como maestro es en tanto se hace responsable de los demás, lo cual implica un descentramiento de sí mismo para pensarse en clave de los otros. En las indagaciones durante los procesos formativos acerca de sus motivaciones para ser maestros, fue interesante la frecuencia de sus respuestas asociadas a un alto grado de compromiso social, y también, a cierta conciencia de la educación como una acción en la que, necesariamente, se afecta a los demás y a sí mismo.

La cotidianidad escolar también contribuye a la complejidad de ser maestros; la tensión entre lo efímero y lo permanente de la escuela, entre lo instantáneo y lo sistemático, entre el acontecimiento y el proceso, entre lo local y lo global, entre lo tradicional y lo moderno, se constituye en lo que define esa cotidianidad escolar. En estos movimientos pendulares que se suceden en el interior de la escuela, les es exigible a los maestros un posicionamiento emocional, moral, cognitivo y afectivo, desde donde puedan tomar distancia del implacable péndulo, y, a la vez, tratar de no ser arroIlados por este intentando hacer su trabajo.

La complejidad del ser maestro, que aún no alcanza a definirse completamente, la tramita cada sujeto como puede y con los recursos de que dispone; los procesos formativos trataron de poner en evidencia estas complejidades, no para trazar caminos o rutas deseables de ser transitadas por los sujetos-maestros, sino para intentar identificarlas, comprenderlas y poner en evidencia para cada uno lo que sucede en cada sujeto al posicionarse en algún punto y de alguna manera. La intencionalidad de ese posicionamiento es, al final, lo que orienta al sujeto en su posibilidad de ser maestro.

Un tercer elemento de los procesos formativos en torno al oficio del maestro fue explorar el carácter pedagógico y educativo de la investigación; es decir, sus posibilidades formativas. Las historias de vida o las narraciones sobre la memoria, más allá de constituirse 
en fuentes primarias o en un medio para alcanzar mejores comprensiones de los fenómenos educativos y pedagógicos, se justifican en sí mismas como formativas: desde la organización para contar, desechar para priorizar y publicar para volver a organizar. Este ejercicio, sin arriesgar rigurosidades de orden metodológico, le apuesta a la posibilidad de trabajar sobre sí mismo, en esas exploraciones acerca de ser maestro.

Entender al sujeto-maestro como eje de la formación continua implicó un reordenamiento a todo lo que atañe a estos procesos, pues se alteró el principio básico sobre el cual, tradicionalmente, se han organizado este tipo de ejercicios: la naturaleza y la procedencia del saber de los maestros. Al comprender que su saber no necesariamente estaría por fuera de ellos, o de sus prácticas o de sus contextos, pero también, dimensionando la importancia de su relación con otros saberes especializados, simplemente, se producía un efecto de deslegitimación de los argumentos que justificaban la formación continua de maestros en términos de ausencia o de déficit.

Estos procesos formativos, generalmente nombrados como capacitación que pretenden hacer capaz a alguien, o como perfeccionamiento ante la supuesta existencia de un estado ideal del ser maestro al cual debería aspirarse, o como cualificación asociada a una perspectiva acumulativa y siempre progresiva del conocimiento que se enseña, ubican al sujeto-maestro en un lugar de dependencia por el saber y el hacer de otros.

Entender la formación continua en torno a la experiencia pedagógica supuso como su eje al maestro, y en torno a él, la definición y la organización de los saberes de la formación, a donde confluían los saberes disciplinares, los saberes que se habían decantado en la práctica, los saberes relacionados con las formas de asumir, y, en ocasiones, apropiar las políticas educativas, con la comprensión de las maneras como aprendían tanto los estudiantes como los otros adultos, con los rituales y las convenciones que regían los comportamientos y las relaciones sociales, con el conocimiento sobre los contextos y los territorios, con las aspiraciones colectivas (legítimas o no), con las formas de gobierno sobre los otros y con los lugares de poder de cada cual.
En este sentido, la experiencia hizo parte de un entramado cultural, urdido por juegos de verdad, poder y formas de relación del sujeto-maestro consigo mismo y con los demás. El maestro, como sujeto de la formación, era el sujeto de la experiencia, y a través de ella se formaba, se transformaba y volvía a tomar forma. La formación era el resultado de la experiencia acumulada en su continuidad y en la posibilidad de hacer propio lo distinto, como conocimiento y como sensibilidad. Era experiencia porque la formación estaba situada en un horizonte de relaciones en el que el reconocimiento de uno mismo se daba en el ser otro, en la interacción, en la conversación.

La palabra y la evocación (es decir, la narración) se constituyeron en las formas como el sujeto producía y se producía a sí mismo; somos lo que nombramos y lo que evocamos. La narración hacía posible, justamente, la creación de los lugares y la organización de los saberes, desde donde se hacían y se originaban a sí mismos los sujetos; es decir, esos lugares que los configuraba, los gobernaba, a su vez, representándolos como tales.

La experiencia de narrar-se empoderó a los sujetos en tanto les permitió detenerse y pensarse, explorar y ordenar, contar y, de modo consecuente, escribir. Lo anterior, mediante un proceso de pensamiento complejo, no solo de naturaleza cognitiva, sino también, sensible y emocional, que atravesaba sus cuerpos. La autobiografía, la historia de vida, la narración sobre el otro o la memoria colectiva se constituyeron en posibilidades de develar ese punto cero en el que se hallaba instalado cada sujeto, y que les permite ver $y$ ocultar. Fue un viaje por sí mismos; tal vez, recorriendo lugares de la memoria ya conocidos, pero deteniéndose donde antes no lo habían hecho, o, también, encontrando recuerdos perdidos. Ese espacio de la experiencia —el pasado- también fue móvil; lo que los sujetos decían sobre el pasado no era acabado, ni permanente ni estable.

La posibilidad de ampliar la mirada de la experiencia necesitó a los sujetos: no solo su propio reconocimiento, sino también, el de los demás. Las potencialidades de la narrativa para la formación docente se incrementaron cuando se compartió lo producido, 
cuando se intentó escuchar genuinamente; es decir, desde lo que realmente pretendió comunicar el otro convocando conversaciones con la intención, especialmente, de comprender lo que se pretendía decir. Narrar-se, ser narrado, ser contado en primera persona o por otros una o varias veces, a través de sí mismo, de la mirada del otro o a propósito de un acontecimiento, se constituyó en la posibilidad de establecer una relación directa entre la experiencia de los maestros, la memoria y la formación docente.

La introducción de las historias de vida en la formación de docentes provocó movilizaciones importantes, como las siguientes:

1. Una movilización en los fines de la formación donde las historias de vida de los maestros permitían develar un sentido estratégico de su enunciación como sujeto, hacia otra perspectiva donde las historias de vida y otras formas de narración permitieron el reconocimiento de los otros como eje de la formación. En este desplazamiento se delineó una ruta formativa importante, a partir de la constitución del sujeto, y donde a la vez que es posible transitar por sí mismo, también es necesario descentrarse de su perspectiva, para reconocerse en los otros. Se advierte una perspectiva de trabajo que se abre hacia la memoria, en tanto las genealogías personales convocan recuerdos que trascienden al sujeto, que se instalan en lo sucedido en la escuela, en el aula y en la ciudad.

2. Una movilización en el tipo de narrativas y en sus finalidades formativas, por cuanto se pasó de las narraciones autobiográficas y biográficas, inicialmente usadas como estrategia de formación que permitían develar espacios y tiempos donde se han producido los maestros, a otros relatos a propósito de sus experiencias en las escuelas o en las aulas, donde el acto de relatar y el de escribir se constituyeron en la formación. Se entendió la narrativa no como el medio para la formación, sino como el acto formativo, en tanto narrar-nos o narrar-los era formar-nos.

3. Una movilización en las perspectivas de trabajo al explorarse los límites de las disciplinas; en especial, de las que han intentado formalizar la producción de las historias de vida, como la antropología, la psicología o la historia; también, identificar otras posibilidades asociadas a la promoción de diferentes diálogos entre ellas, o hacia la generación de miradas críticas a sus discursos y sus prácticas asociadas a su carácter "científico". Mientras más se profundizaban las posibilidades formativas de las genealogías personales de los maestros, más se debilitaban las intencionalidades de su uso para la investigación en educación o pedagogía, y también, las exigencias relacionadas con el rigor metodológico para su producción.

4. Una movilización en torno a las intencionalidades del proceso formativo de los maestros, pues se pasó de objetivos centrados en la construcción de la consciencia histórica del maestro (heredados, seguramente, de la perspectiva disciplinar de este primer proceso formativo) a propósitos asociados a su visibilización como sujeto social y de producción de conocimiento.

5. Una movilización asociada a los objetos de formación, pues la primera experiencia se instaló en un saber curricular, como la historia, y en las posibilidades de su enseñanza, mientras que la segunda experiencia giró en torno a otros saberes asociados a las formas de ser maestros y sus relaciones con las escuelas.

6. Una movilización en las categorías en torno a las cuales se organizó la perspectiva formativa, pues si bien ambos procesos giraron en torno al maestro, la primera acogió la categoría identidad con un carácter relacional, asociada a los procesos que hace el individuo para hacerse maestro, desde una fuerte prescripción externa que expresa cuanto se considera deseable social y políticamente en torno a la educación, y, por supuesto, al maestro; mientras, el segundo proceso formativo se organizó en torno a la subjetividad del maestro, lo cual si bien hace referencia a la manera como se produce este sujeto, a la vez, puede generar fracturas a todo lo que se demanda desde el afuera: un maestro-investigador, un maestro-intelectual, un maestro-planeador, un maestro-competente, un maestro-profesional, un maestro-productivo. 


\section{Referencias}

Alliaud, A. y Suárez, D. H. (2011). El saber de la experiencia: narrativa, investigación y formación docente. CLACSO. Facultad de Filosofía y Letras de la Universidad de Buenos Aires.

Campos, H. (2012). La recuperación de semióticas en las historias de vida para la reflexión y mejora de las prácticas escolares. En: J. Rivas., F. Hernández, J. Sancho y C. Núñez (Coords.). Historias de vida en educación: sujeto, diálogo, experiencia. REUNID (Red Universitaria de Investigación e Innovación Educativa)-Universidad de Barcelona-Esbrina-PROCLE (pp. 93-98). Barcelona, España. Recuperado de: http://hdl. handle.net/2445/32345

Caparrós, E. y Sierra, E. (2012). Al hilo de lo vivido: la pedagogía como brújula para la investigación. En: J. Rivas, F. Hernández, J. Sancho y C. Núñez (Coords.). Historias de vida en educación: sujeto, diálogo, experiencia. REUNID (Red Universitaria de Investigación e Innovación Educativa)-Universidad de BarcelonaEsbrina-PROCLE (pp. 61-66). Barcelona, España. Recuperado de: http://hdl.handle.net/2445/32345

Clavijo, A. (2000). Formación de maestros, historia y vida. Reflexión y praxis del maestro acerca de la lectura y la escritura. Bogotá, Colombia: Universidad Distrital, Plaza y Janés.

Goodson, I. (1981). Life history and study of schooling. Interchange on educational Policy 11,(4), 62-76. Ontario, Canadá.

Goodson, I. (1994). Studying the teachers' lives. Professional Life and Work. Gran Bretaña: Routledge.
Goodson, I. (2004). Historias de vida del profesorado. Barcelona, España: Octaedro.

González, M., Jiménez, A., Neira, A y Cortes, A. (2015). Entre la historia y la memoria: alternativas en la formación de maestros. Bogotá, Colombia: IDEP. Documento mimeo.

Knowles, J. (2005). Modelos para la comprensión de las biografías del profesorado en formación y en sus primeros años de docencia. En: Historias de vida del profesorado. Barcelona, España: Octaedro.

Meirieu, P. (1998). Frankenstein educador. Barcelona: Laertes.

Mélich, J. (2012). Filosofía de la Finitud. Barcelona: Herder, $2^{\mathrm{a}}$ edición.

Palou, J. y Fons, M. (2012). El yo y el nosotros en la configuración de las experiencias: espacio y personajes en los relatos de vida lingüística. En: J. Rivas, F. Hernández, J. Sancho y C. Núñez(Coords.). Historias de vida en educación: sujeto, diálogo, experiencia. REUNID, (Red Universitaria de Investigación e Innovación Educativa-Universidad de Barcelona)-EsbrinaPROCLE. Barcelona, España. Recuperado de: http://hdl.handle. net/2445/32345

Ricoeur, P. (2006). La vida: un relato en busca de narrador. Revista Ágora. Papeles de Filosofía 25, 9-22.

White, H. (1981). Metahistoria: La imaginación histórica en el siglo xIx. México: Fondo de Cultura Económica.

Zemelman, H. (1998). Sujeto: Existencia y potencia. México: Anthropos, Centro Regional de Investigaciones Multidisciplinarias (UNAM). 


\section{Diálogo del conocimiento}

\section{Entre narratividad y alteridad}

¿Dónde ha quedado el observador? ¿El narrador? ¿El poeta en un mundo ya repartido? ¿El que describe? ¿El que emplea la distinción para diferenciar y designar algo? Aquel al que se podía preguntar: ¿Por qué así, por qué no de otra manera?

Niklas Luhman

Al leer el artículo Narrar_nos es formar_nos: las historias de vida en la formación de maestros, de Mireya González Lara, encuentro varios elementos interesantes, los cuales me gustaría contrastar con el documento de Alfonso Mendiola El giro historiográfico: la observación de observaciones del pasado. Para hacerlo, empiezo retomando a Michael Baxandall, historiador de pintura, quien para dar cuenta de los problemas que trabaja argumenta que él no explica cuadros, sino que, explica observaciones sobre cuadros; en mi caso, planteo que el diálogo será trabajado a partir de las posibilidades de la interpretación de los elementos subjetivos y narrativos del documento; parafraseando al autor: la intención es de alguna manera estudiar las posibilidades de comunicación alrededor de las narrativas.

Sin duda alguna trabajar narrativas, ya sea como categoría teórica o metodológica, permite un sinnúmero de posibilidades para dar a luz elementos constitutivos y esenciales del ser de cada sujeto. Por otro lado, las historias de vida permiten reconocer una situación en relación con la construcción de un sujeto o varios de ellos, siempre puestos en un foco en común, es presuponer precisamente que la vida es una historia (Bordieu, 1997). Por ello, siempre es importante poner en discusión las posibilidades y variedad de los elementos narrativos y las construcciones de historias de vida, ya que las narrativas solo son espacios profundos en las historias de vida.

En ese sentido, la discusión se debe entablar sobre la siguiente concepción epistemológica: la hermenéutica y el renacimiento, ya que esa etapa en la historia occidental dio lugar a unas forma de pensamientos y transformaciones frente a lo que significó el largo letargo de la edad media; se relacionan con varios eventos que generaron un giro en la forma de ver y entender el mundo, verbigracia la Reforma Protestante, en este punto es clave Martín Lutero quien es protagonista de muchos de los cambios "humanistas" en la Europa occidental. A través de las tesis que propuso se funda la religión protestante, la cual tiene como eje el "Libre examen", esta tesis dota al ser humano de entendimiento e inteligencia para leer la Biblia y, en una aproximación a esta contemporaneidad, leer los textos que se hacen sagrados; en un nivel más profundo, leer los contextos no solo del otro sino los propios y dar cuenta de las relaciones y hechos sociales que allí se dan. Retomando la idea de Lutero, implica también tener un libro sagrado traducido a lenguas vernáculas y la fundación del idioma alemán como elemento de cohesión de los pueblos germánicos.

En sí mismo este giro de comprensión del mundo marca de alguna manera el fin del monopolio cristiano sobre la orientación moral de los feligreses; es, precisamente, el reconocimiento de la capacidad de la razón humana para conocer. En ese sentido, y en palabras del maestro Luis Fernando Marín, a partir de lecturas muy rigurosas sobre la teoría del poder en Foucault: "se inaugura la época de la libertad para los europeos". Algo similar se pretende con estas nuevas metodologías que rompen con los esquemas clásicos de las ciencias sociales y es poder leer_nos y generar_nos conocimientos, saberes a partir de construcciones sociales y culturales propias; en otras palabras: inaugurar una época de libertad para los no europeos.

114 nodos y nudos / volumen 4 N. ${ }^{\circ} 40$ / enero - junio de 2016 /ISSN: 0122-4328/pp. 103-116/ 
La hermenéutica ya viene constituyendo un giro en las formas de aprehender el mundo y sus efectos van a recaer en la cultura, más aún, los procesos de enseñanza aprendizaje van a ser los ejes clave para las reformas liberales a través del desciframiento de textos antes exclusivos para ciertas elites; esto recibirá el nombre de secularización del conocimiento. Lo que en ese punto de la historia se jugó, fue la posibilidad de criticar a partir de juicios razonables las verdades reveladas que marcaron la visión de mundo en la edad media. De ahí que sea posible llegar a una definición de la hermenéutica donde el comprender es explorar al sujeto; es una forma de colocarlo en el lugar del otro, es ir más allá de lo simple y evidente.

Teniendo en cuenta lo anterior, una de las grandes estrategias para llegar a comprender de alguna manera ese lugar del otro, es a través de las narrativas, nos comunicamos, pero no solo damos a conocer datos, narramos vidas, hechos, sentimientos y pensamientos, los hacemos experiencias y los validamos en prácticas comunes. De otra parte, las historias de vida nos permiten enfocarnos en un punto y conocer las diversas posturas de una manera profunda, estableciendo algunas relaciones de tipo social, económico y/o cultural. La historia de vida permite unir elementos históricos de una persona y su relación con otro tipo de hechos en distintos niveles de análisis, por ende, los datos de los sujetos son más del tipo social y es posible que se nos pierdan algunas características donde nos aproximemos más al sujeto y no tanto al objeto, sin embargo, explorar a profundidad la vida y narrativas nos pone en un lugar literario. Bourdieu lo interpreta como "una vida es inseparablemente el conjunto de los acontecimientos de una existencia individual concebida como una historia y el relato de esa historia" (Bordieu, 1997, pág. 121) a partir de la novela Une Vie, ya que lo que hace el autor es llevarnos por un viaje a través de Normandía como si el lector fuera al lado de Jeanne su protagonista, es una construcción de una vida a partir de un contexto y una secuencia de acontecimientos (Maupassant, 2006).

La cultura se presenta a sí misma a través de relatos narrativamente controvertidos debido a dos razones principales. Primero, las acciones y las relaciones humanas se forman según una doble hermenéutica: identificamos lo que hacemos por medio de un relato de lo que hacemos. Las palabras y los hechos son equiprimoridales, en el sentido de que casi toda acción humana socialmente significativa más allá de rascarse la nariz, se identifica como una cierta clase de hacer a través de los relatos que tanto los propios agentes como los demás dan de ese hacer. La segunda razón por la que la cultura se presenta a sí misma a través de relatos controvertidos es que no sólo las acciones e interacciones humanas están constituidas por relatos, que en conjunto forman una "red de relatos", sino que también están constituidas por la postura valorativa de los actores hacia lo que hacen. (Benhabib, 2006, pág. 31).

De esta manera es que narrar_nos es encontrar_nos en un plano distinto al que el libre mercado nos impone a diario, para narrar_nos necesitamos poner a todo vapor la locomotora de la imaginación y constituir_nos identidades que posibiliten hacer un giro educativo, en el cual evitemos reducir o escencializar a los sujetos de la educación, con el fin de reconocer_nos como iguales a partir de las diferencias que constituyen un mejor ser, uno para quien la narratividad se hace alteridad. Las narrativas hacen a los sujetos, las narrativas no se construyen a partir del sujeto individual, sino de la trascendencia donde hay un conjunto de identificaciones con el otro (la cultura), para reivindicar una identidad del sujeto. Para finalizar, y en palabras de Paul Ricoeur, la identidad es alcanzada por una mediación narrativa.

Iván Martínez Triana 


\section{Referencias}

Benhabib, S. (2006). Las reivindicaciones de la cultura. Igualdad y diversidad en la era global. Buenos Aires: Katz.

Bordieu, P. (1997). Razones prácticas: sobre la teoría de la acción. Barcelona: Anagrama.

Maupassant, G. d. (2006). Una vida. Barcelona: Debolsillo.

Mendiola, A. (2000). El giro historiográfico: la observación de observaciones del pasado. Historia y Grafía (15), 181-208.

Ricœur, P. (s.f.). La identidad narrativa (pp. 339-335). Recuperado de https://textosontologia.files.wordpress. com/2012/09/identidad-narrativa-paul-ricoeur.pdf

116 nodos y nudos / volumen 4 N. ${ }^{\circ} 40$ / enero - junio de 2016/ISSN: 0122-4328/pp. 103-116/ 